\title{
CONGRUENCE QUASI-ORDERABILITY IN SUBTRACTIVE VARIETIES
}

\author{
PAOLO AGLIANO
}

(Received 12 March 1999; revised 21 September 2000)

Communicated by B. A. Davey

\begin{abstract}
In this paper we investigate subtractive varieties of algebras that are congruence quasi-orderable. Though this concept has its origin in abstract algebraic logic, it seems to be worth investigating in a purely algebraic fashion. Besides clarifying the algebraic meaning of this notion, we obtain several structure theorems about such varieties. Also several examples are provided to illustrate the theory.
\end{abstract}

2000 Mathematics subject classification: primary 08A99, $08 \mathrm{~A} 30$.

Keywords and phrases: subtractive variety, quasi-orderability.

\section{Introduction}

In this paper we continue the investigation, started in [1], on the algebraic consequences of the so called Fregean axiom. Originally this axiom was considered in the context of a very specific logical system, that was proposed by Suszko for formalizing the logic underlying Wittgenstein's Tractatus. Independently, Büchi and Owens considered a purely algebraic notion [9]. Later Pigozzi recognized the connections between the two notions and began the investigation of what we call now congruence orderable varieties; in [19] he showed that every point-regular congruence orderable variety is equivalent to a variety of Skolem lattices with compatible operations. This was the first representation theorem of this kind; later Idziak et al. [15] proved that every point-regular congruence permutable congruence orderable variety is equivalent to a variety of equivalential algebras with compatible operations. They also showed that a point-regular congruence orderable variety is strongly point-regular if and only if it is arithmetical; in this way they provided an alternative formulation of the results

(c) 2001 Australian Mathematical Society 0263-6115/2001 \$A2.00+0.00 
in [19]. Our contribution to this series is in [1]; there we have shown that a subtractive variety with definable principal ideals is equivalent to a variety of Hilbert algebras with compatible operations.

At the same time closely related results were being obtained in a logic context; discussing them in details would constitute a detour from the matter at hand, but we refer the reader to $[10,11,12,13]$.

In this paper we take this investigation in yet another direction, by considering the more general class of congruence quasi-orderable varieties. This concept arises naturally if one works in the context of ideal theory, which originated with Ursini [20] and has been developed in $[3,4,5]$. Combining the techniques already used in $[1,15,19]$ with the methods of ideal theory we obtain a number of analogs of the representation theorems mentioned above. More specifically Theorem 3.6 is a (partial) generalization of the results in [15], Theorem 4.2 is a (partial) generalization for the results in [1] and Theorem 4.13 is a (partial) generalization for the results in [19].

It is not yet clear if the notion of congruence-orderability has a logical meaning, nor if the assumption of subtractivity (which we will make throughout the paper) is absolutely necessary. It is certainly crucial in all our proofs, but we cannot exclude the possibility of replacing it with a weaker concept.

We follow more or less the standard notation of general algebra; in particular if $\mathbf{A}$ is an algebra, then Con(A) denotes its congruence lattice and $1_{A}$ and $0_{A}$ denote the largest and smallest elements of $\operatorname{Con}(\mathbf{A})$. If $X \subseteq A^{2}$, then $\vartheta_{\mathbf{A}}(X)$ is the smallest congruence containing all pairs in $X$ and if $X=\{a, b\}$ we write $\vartheta_{\mathbf{A}}(a, b)$. For $\theta \in \operatorname{Con}(\mathbf{A})$ and $(a, b) \in \theta$, sometimes we use the relational notation ' $a \theta b$ '. If $X \subseteq A^{2}$ and $a \in A$, then $a / X=\{(b:(a, b) \in X\}$; hence if $\theta \in \operatorname{Con}(\mathbf{A})$ and $a \in A$, then $a / \theta$ is the congruence class of $a$. Finally, if $Y \subseteq A, \operatorname{Sub}_{\mathbf{A}}(Y)$ is the smallest subalgebra of $\mathbf{A}$ containing $Y$.

A variety $\mathscr{V}$ is pointed if it has a constant, which will be denoted by 0 , in its type. On each algebra $\mathbf{A} \in \mathscr{V}$ one can consider the quasi-order $\preccurlyeq_{\mathbf{A}}$ : for $a, b \in A$,

$$
a \preccurlyeq_{A} b \text { if and only if } \vartheta_{A}(0, a) \subseteq \vartheta_{A}(0, b) \text {. }
$$

If $\approx_{\mathbf{A}}=0_{\mathrm{A}}$, then the algebra $\mathbf{A}$ is said to be congruence orderable; if this happen throughout a class $\mathscr{K}$ of algebras, then the class is congruence orderable $[1,15]$. The reason for the name is that the quasi-ordering $\preccurlyeq_{A}$ becomes a partial ordering in this case. We shall deal with a weaker notion: an algebra $\mathbf{A}$ in a pointed variety is congruence quasi-orderable if $\approx_{\mathbf{A}} \in \operatorname{Con}(\mathbf{A})$. Likewise a class $\mathscr{K}$ of algebras is congruence quasi-orderable if each of its members is congruence quasi-orderable. Let us start with an easy fact about $\approx_{\mathrm{A}}$. 
PROPOSITION 1.1. For any pointed algebra $\mathbf{A}$ and any $\alpha \in \operatorname{Con}(\mathbf{A})$ we have

$$
\left(\approx_{\mathbf{A}} \vee \alpha\right) / \alpha \subseteq \approx_{\mathbf{A} / \alpha},
$$

where the join is evaluated in the lattice of equivalence relations.

Proof. Choose $a, b \in A$; it is obvious that if $a \preccurlyeq_{A} b$, then $a / \alpha \preccurlyeq_{\mathrm{A} / \alpha} b / \alpha$ for each $\alpha \in \operatorname{Con}(\mathbf{A})$. Suppose now that $(a / \alpha, b / \alpha) \in\left(\approx_{\mathbf{A}} \vee \alpha\right) / \alpha$. The pair $(a, b)$ lies in the transitive closure of $\alpha \circ \approx_{\mathrm{A}}$, that is contained in the transitive closure of $\alpha \circ \preccurlyeq_{\mathrm{A}}$. Hence there is a chain of elements of $A, a=c_{0}, c_{1}, \ldots, c_{n}=b$ such that for every $i$ either $\left(c_{i}, c_{i+1}\right) \in \alpha$ or $c_{i} \preccurlyeq_{\mathrm{A}} c_{i+1}$. Factoring by $\alpha$, either $c_{i} / \alpha=c_{i+1} / \alpha$ or $c_{i} / \alpha \preccurlyeq_{\mathrm{A} / \alpha} c_{i+1} / \alpha$. Hence $a / \alpha=c_{0} / \alpha \preccurlyeq_{\mathrm{A} / \alpha} c_{n} / \alpha=b / \alpha$. By symmetry we conclude that $(a / \alpha, b / \alpha) \in \approx_{\mathbf{A} / \alpha}$.

Unfortunately, in general, the inequality in Proposition 1.1 remains strict even if $\mathbf{A}$ is congruence quasi-orderable. In particular, it might happen that $\mathbf{A}$ is congruence quasi-orderable and $\approx_{A / \approx_{A}}>0_{A / \approx_{A}}$, a characteristic we would like to avoid. For this (and other) reason we restrict our attention to subtractive varieties, a move that has already been proven useful when dealing with congruence orderable varieties [1]. We postpone the definition of subtractive variety to the next section; however we would like to point out the following facts.

- A subtractive congruence orderable-variety is point regular, hence Fregean ([1, Theorem 2.1]).

- There are congruence orderable varieties that fail to be subtractive ([1, Example 2.3]).

- There are subtractive varieties that are congruence quasi-orderable but not congruence orderable (Proposition 4.4).

- There are subtractive varieties that fail to be congruence quasi-orderable (Example 2.10).

- If $\mathbf{A}$ belongs to a congruence quasi-orderable subtractive variety, then $\approx_{\mathbf{A} / \approx_{A}}=$ $0_{A / \approx_{A}}$. Hence $A / \approx_{A}$ is congruence orderable (Corollary 2.2).

- The class of congruence orderable algebras in a congruence quasi-orderable variety plays a very important role.

\section{Characterizing subtractive congruence quasi-orderable varieties}

Subtractive varieties have been widely investigated in the past years (see for instance [5] and the extensive bibliography therein). If $\mathscr{K}$ is a class of similar pointed algebras, a term $p\left(x_{1}, \ldots, x_{m}, y_{1}, \ldots, y_{n}\right)$ is a $\mathscr{X}$-ideal term in $\vec{y}$ (and we write $p(\vec{x}, \vec{y}) \in$ $\left.\mathrm{IT}_{\mathscr{X}}(\vec{y})\right)$ if the identity $p(\vec{x}, 0, \ldots, 0) \approx 0$ holds in $\mathscr{X}$. A nonempty subset $I$ of 
$\mathbf{A} \in \mathscr{K}$ is a $\mathscr{K}$-ideal of $\mathbf{A}$ if for any $p(\vec{x}, \vec{y}) \in \operatorname{IT}_{\mathscr{X}}(\vec{y})$, for $\vec{a} \in A$ and $\vec{b} \in I$, $p(\vec{a}, \vec{b}) \in I$. Under inclusion, the set $\mathrm{I}_{\mathscr{X}}(\mathbf{A})$ of all $\mathscr{X}$-ideals of $\mathbf{A}$ is an algebraic lattice. If $H \subseteq A$, the ideal $\langle H\rangle_{A}^{\mathscr{X}}$ generated by $H$ is the $\operatorname{set}\{p(\vec{a}, \vec{b}): p(\vec{x}, \vec{y}) \in$ IT $\left._{\mathscr{X}}(\vec{y}), \vec{a} \in A, \vec{b} \in H\right\}$. In contrast with the previous notation we shall denote by $(a)_{\mathbf{A}}^{\mathscr{K}}$ the principal $\mathscr{K}$-ideal generated by $\{a\}$. Note that $(0)_{\mathbf{A}}^{\mathscr{X}}=\{0\}$. When $\mathscr{K}$ is $\{\mathbf{A}\}$ (or, equivalently, the variety $\vee(\mathbf{A})$ generated by $\mathbf{A}$ ), then a $\mathscr{K}$-ideal is called an ideal and we drop all the affixes and suffixes in sight. Using just the definition of ideal term one sees easily that, for any reflexive subalgebra $\mathbf{S}$ of $\mathbf{A} \times \mathbf{A}, 0 / S=\{a:(0, a) \in S\}$ is an ideal of $\mathbf{A}$. Thus $0 / \theta \in \mathrm{I}(\mathbf{A})$ for any $\theta \in \operatorname{Con}(\mathbf{A})$.

A class of pointed algebras $\mathscr{K}$ is subtractive if for some binary term $s(x, y)$

$$
s(x, 0) \approx x, \quad s(x, x) \approx 0
$$

hold in $\mathscr{K}$. In the sequel any term satisfying the above equation will be called informally a witness term or a term witnessing subtractivity for $\mathscr{V}$. Several characterizations of subtractive varieties can be found in $[2,3,20]$. Here we recall that the following are equivalent:

(1) $\mathscr{V}$ is subtractive.

(2) The congruences of each algebra in $\mathscr{V}$ permute at 0 , that is for any $\mathbf{A} \in \mathscr{V}$, $\theta, \varphi \in \operatorname{Con}(\mathbf{A})$ and $a \in A$ if $(0, a) \in \theta \circ \varphi$ then $(0, a) \in \varphi \circ \theta$.

(3) For all $\mathbf{A} \in \mathscr{V}$, the mapping 0/ is a lattice epimorphism from $\operatorname{Con}(\mathbf{A})$ into I(A). It is a nontrivial fact [20, Proposition 1.4] that in a subtractive variety $\mathscr{V}, \mathrm{I}_{\mathscr{V}}(\mathrm{A})=\mathrm{I}(\mathrm{A})$ for each member $\mathbf{A} \in \mathscr{V}$. Thus if $\mathbf{A}$ belongs to a subtractive variety $\mathscr{V}$ we shall denote the lattice of ideals of $\mathbf{A}$ simply by $\mathrm{I}(\mathbf{A})$, without any reference to $\mathscr{V}$.

If a subtractive variety $\mathscr{V}$ happens to be also (congruence) point regular, then the mapping $\theta \mapsto 0 / \theta$ is an isomorphism from $\operatorname{Con}(\mathbf{A})$ to $\mathrm{I}(\mathbf{A})$, for each $\mathbf{A} \in \mathscr{V}$. In this case the variety is said to be ideal-determined (groups, rings, Boolean algebras, vector spaces etc. form varieties of this kind). Any subtractive congruence orderable variety is ideal-determined [1, Theorem 2.1].

We say that a pointed algebra is subtractive if it generates a subtractive variety. The third fact above implies that each ideal of a subtractive algebra $A$ is $0 / \theta$ for some $\theta \in \operatorname{Con}(\mathbf{A})$; in particular we must have, for each $a \in A, 0 / \vartheta_{\mathbf{A}}(0, a)=(a)_{\mathrm{A}}$. This allows us to express congruence quasi-orderability solely in terms of principal ideals. Since for any algebra $\mathbf{A}$ and $u, v \in A$,

$$
\vartheta_{\mathbf{A}}(u, v)=\vartheta_{\mathbf{A}}\left(\{u\} \times u / \vartheta_{\mathbf{A}}(u, v)\right),
$$

if $\mathbf{A}$ is subtractive, taking $u=0$ and $v=a$ or $v=b$ we get at once

$$
a \approx_{\mathrm{A}} b \text { if and only if }(a)_{\mathrm{A}}=(b)_{\mathrm{A}} .
$$

The fact that in a subtractive variety ideals can be always described via (ideal) terms is a powerful tool for investigating the congruence $\approx_{A}$. 
PROPOSITION 2.1 ([3]). Let $\mathbf{A}$ be a subtractive algebra, let $h: \mathbf{A} \rightarrow \mathbf{B}$ be an onto homomorphism and let $X \subseteq A$. Then for any $a \in A$,

$$
h(a) \in\langle h(X)\rangle_{\mathbf{B}} \text { if and only if } a \in\langle X\rangle_{\mathbf{A}} \vee 0 / \operatorname{ker} h .
$$

COROLlaRY 2.2. Let $\mathbf{A}$ be a subtractive algebra. For each $\alpha \in \operatorname{Con}(\mathbf{A})$ and $a, b \in A$

$$
a / \alpha \approx_{\mathbf{A} / \alpha} b / \alpha \text { if and only if }(a)_{\mathbf{A}} \vee 0 / \alpha=(b)_{\mathbf{A}} \vee 0 / \alpha
$$

Hence if $\mathbf{A}$ is congruence quasi-orderable, then $\approx_{\mathbf{A} / \approx_{A}}=0_{\mathbf{A} / \approx_{A}}$ and $\mathbf{A} / \approx_{\mathbf{A}}$ is congruence orderable.

PROOF. By definition $a / \alpha \preccurlyeq_{\mathbf{A} / \alpha} b / \alpha$ if and only if $a / \alpha \in(b / \alpha)_{\mathbf{A} / \alpha}$. If $\pi$ is the canonical epimorphism from $\mathbf{A}$ to $\mathbf{A} / \alpha$, this is equivalent to say that $\pi(a) \in(\pi(b))_{\mathbf{A} / \alpha}$ and, by Proposition 2.1, this happens if and only if $a \in(b)_{\mathbf{A}} \vee 0 / \alpha$.

For the second fact, observe that $0 / \approx_{A}=\{0\}=(0)_{A}$. Then $a / \approx_{A} \approx_{A / \approx_{A}} b / \approx_{A}$ if and only if $a \approx_{\mathrm{A}} b$ if and only if $a / \approx_{\mathrm{A}}=b / \approx_{\mathrm{A}}$.

The description $\approx_{\mathbf{A} / \alpha}$ above seems at first very encouraging. In particular it looks like we might be able to transfer congruence quasi-orderability from an algebra to its homomorphic images. This point, however, is not very clear yet and it will be discussed some at the end of this section. Meanwhile we shall offer two different characterizations of congruence quasi-orderable subtractive varieties. The first is based on the investigations in $[3,4]$ of the relationships between ideals and congruences in subtractive algebras.

For any pointed algebra $\mathbf{A}$ and $I \in \mathbf{I}(\mathbf{A})$ one may consider the congruence

$$
I^{\varepsilon}=\bigvee\{\theta: 0 / \theta=I\} .
$$

This congruence has been studied in detail in [4] where the following lemma is proved implicitly.

LEMMA 2.3. Let $\mathbf{A}$ be subtractive. Then for any $I \in \mathrm{I}(\mathbf{A})$

$$
I^{\varepsilon}=\left\{(a, b): \text { for all } p \in \operatorname{Pol}_{1}(\mathbf{A}), p(a) \in I \text { if and only if } p(b) \in I\right\} .
$$

LEMMA 2.4. Let $\mathbf{A}$ be subtractive with witness term $s(x, y)$. Then

$$
(0)_{\mathrm{A}}^{\varepsilon} \subseteq\{(a, b): s(a, b)=s(b, a)=0\} \subseteq \approx_{\mathrm{A}} .
$$

Moreover, $(0)_{\mathrm{A}}^{\varepsilon}$ is the largest congruence contained in $\approx_{\mathrm{A}}$. 
Proof. To see that $(0)_{\mathrm{A}}^{\varepsilon} \subseteq\{(a, b): s(a, b)=s(b, a)=0\}$ apply Lemma 2.3 with $I=(0)_{\mathrm{A}}, p(x)=s(a, x)$ and $q(x)=s(x, b)$. For the other inclusion we prove a stronger statement: If $\mathbf{A}$ is subtractive and $s(x, y)$ is any subtraction term, then for all $a, b \in A$,

$$
a \approx_{\mathrm{A}} b \text { if and only if } s(a, b), s(b, a) \in(a)_{\mathbf{A}} \cap(b)_{\mathbf{A}} .
$$

If $a \approx_{\mathrm{A}} b$, then $s(a, b), s(b, a) \in(a)_{\mathrm{A}}=(a)_{\mathrm{A}} \cap(b)_{\mathrm{A}}$, since $s(x, y)$ is an ideal term in $x, y$.

Conversely assume that $s(a, b), s(b, a) \in(a)_{\mathbf{A}} \cap(b)_{\mathbf{A}}$. Let $u(x, y, z)=s(x$, $s(s(x, y), z))$; it is easily checked that $u$ is an ideal term in $y, z$, hence

$$
a=u(a, b, s(a, b)) \in(b)_{\mathrm{A}}, \quad b=u(b, a, s(b, a)) \in(a)_{\mathrm{A}}
$$

and therefore $a \approx_{\mathrm{A}} b$.

Finally, if $\theta \subseteq \approx_{\mathrm{A}}$, then $0 / \theta=0 / \approx_{\mathrm{A}}=\{0\}$. Thus $\theta \subseteq(0)_{\mathrm{A}}^{\varepsilon}$.

COROLlARY 2.5. Let $\mathbf{A}$ be a subtractive algebra. Then $\mathbf{A}$ is congruence quasiorderable if and only if for any binary term $s(x, y)$ witnessing subtractivity for $\mathbf{A}$ one has

$$
\approx_{\mathrm{A}}=\{(a, b): s(a, b)=\stackrel{s}{s}(b, a)=0\}=(0)_{\mathrm{A}}^{\varepsilon} .
$$

COROLlARY 2.6. Let $\mathbf{A}$ be subtractive and suppose that $\mathbf{A} / \theta$ is congruence quasiorderable for some $\theta \in \operatorname{Con}(\mathbf{A})$. Then

$$
(0 / \theta)^{\varepsilon}=\{(a, b): s(a, b), s(b, a) \in 0 / \theta\} .
$$

PROOF. The proof is based on the following fact, whose proof is straightforward. If $h: \mathbf{A} \rightarrow \mathbf{B}$ is an epimorphism and $I \in \mathrm{I}(\mathbf{B})$, then for any $a, b \in A$

$$
(a, b) \in\left(h^{-1}(I)\right)_{\mathrm{A}}^{\varepsilon} \quad \text { if and only if } \quad(h(a), h(b)) \in I^{\varepsilon} .
$$

Let $h$ be the natural epimorphism from $\mathbf{A}$ onto $\mathbf{A} / \theta$. By Corollary 2.5 applied to $\mathbf{A} / \theta$ we get

$$
\begin{array}{rll}
(a, b) \in(0 / \theta)^{\varepsilon}=\left(h^{-1}(0)\right)_{\mathbf{A}}^{\varepsilon} & \text { if and only if } & (h(a), h(b)) \in(0)_{\mathbf{A} / \theta}^{\varepsilon} \\
& \text { if and only if } & h(s(a, b)), h(s(b, a))=0 \\
& \text { if and only if } & s(a, b), s(b, a) \in h^{-1}(0)=0 / \theta .
\end{array}
$$

COROLlaRY 2.7. Let $\mathbf{A}$ be a subtractive algebra, $s(x, y)$ be any term witnessing subtractivity for $\mathbf{A}, a, b \in A$ and $I=\langle s(a, b), s(b, a)\rangle_{\mathbf{A}}$. If $\mathbf{A} / I^{\varepsilon}$ is congruence quasi-orderable, then $0 / \vartheta_{\mathbf{A}}(a, b)=I$. In particular, the latter is a compact element in $\mathrm{I}(\mathbf{A})$. 
PROOF. It is obvious that $I \subseteq 0 / \vartheta_{\mathrm{A}}(a, b)$ in any case. For the converse, $(a, b) \in I^{\varepsilon}$ and by Corollary 2.6 we have that $s(a, b), s(b, a) \in I$ and thus $\vartheta_{\mathrm{A}}(a, b) \subseteq I^{\varepsilon}$. Hence $0 / \vartheta_{\mathbf{A}}(a, b) \subseteq I$ and in fact equality holds.

Let $\mathbf{A}$ be a subtractive algebra, $s(x, y)$ a subtraction term for $\mathbf{A}$ and let $I \in \mathrm{I}(\mathbf{A})$. Consider the term $u(x, y, z)$ defined in the proof of Lemma $2.4 ; u(x, y, z)$ is an ideal term and, for any $a, b \in A, a=u(a, b, s(a, b)$. It follows at once that $a \in(a)_{\mathbf{A}} \vee\left(s(a, b)_{\mathbf{A}}\right.$ and $b \in(a)_{\mathbf{A}} \vee(s(b, a))_{\mathbf{A}}$. Thus if $0 / \vartheta_{\mathbf{A}}(a, b) \subseteq I$, then $(a)_{\mathbf{A}} \vee I=(b)_{\mathbf{A}} \vee I$.

PROPOSITION 2.8. Let $\mathbf{A}$ be a subtractive algebra and $\alpha \in \operatorname{Con}(\mathbf{A})$. If for any $a, b \in A$

$$
(a)_{\mathrm{A}} \vee 0 / \alpha=(b)_{\mathrm{A}} \vee 0 / \alpha \text { implies } 0 / \vartheta_{\mathrm{A}}(a, b) \subseteq 0 / \alpha,
$$

then $\mathbf{A} / \alpha$ is congruence quasi-orderable.

ProOf. Let $a / \alpha, b / \alpha \in \mathbf{A} / \alpha$ with $a / \alpha \approx_{\mathbf{A} / \alpha} b / \alpha$. By Corollary 2.2 this implies $(a)_{\mathbf{A}} \vee 0 / \alpha=(b)_{\mathbf{A}} \vee 0 / \alpha$ and thus $0 / \vartheta_{\mathbf{A}}(a, b) \subseteq 0 / \alpha$. If $p(x) \in \operatorname{Pol}_{1}(\mathbf{A})$, then $s(p(a), p(b)), s(p(b), p(a)) \in 0 / \vartheta_{\mathbf{A}}(a, b)$ and hence $s(p(a), p(b)), s(p(b), p(a)) \in$ $0 / \alpha$. If $p(a) \in 0 / \alpha$, then

$$
p(b)=s(p(b), 0)=s(p(b), p(a)) \alpha 0
$$

and similarly if $p(b) \in 0 / \alpha$, then $p(a) \in 0 / \alpha$. By Lemma $2.3(a / \alpha, b / \alpha) \in(0 / \alpha)_{\mathbf{A} / \alpha}^{\varepsilon}$ and by Lemma 2.4 we conclude that $(0 / \alpha)_{\mathbf{A} / \alpha}^{\varepsilon}=\approx_{\mathbf{A} / \alpha}$ and $\mathrm{A} / \alpha$ is congruence quasiorderable.

We are now ready to present the first characterization of congruence quasi-orderable subtractive varieties.

THEOREM 2.9. For any subtractive variety $\mathscr{V}$ the following are equivalent:

(1) $\mathscr{V}$ is congruence quasi-orderable.

(2) For each $\mathbf{A} \in \mathscr{V}, I \in \mathrm{I}(\mathbf{A})$ and $a, b \in A$

$$
(a)_{\mathrm{A}} \vee I=(b)_{\mathrm{A}} \vee I \text { implies } 0 / \vartheta_{\mathrm{A}}(a, b) \subseteq I .
$$

PROOF. Assume that $\mathscr{V}$ is congruence quasi-orderable. If $s(x, y)$ witnesses subtractivity for $\mathscr{V}$, then $0 / \vartheta_{\mathrm{A}}(a, b)=\left\langle s(a, b),(s(b, a)\rangle_{\mathrm{A}}\right.$ by Corollary 2.7 and congruence quasi-orderability. Suppose that $(a)_{\mathrm{A}} \vee I=(b)_{\mathrm{A}} \vee I$ and let $I=0 / \alpha$. By Corollary $2.2(a / \alpha)_{\mathbf{A} / \alpha}=(b / \alpha)_{\mathbf{A} / \alpha}$, thus $s(a, b) / \alpha=0 / \alpha$ by Corollary 2.5. It follows that $s(a, b) \in 0 / \alpha=I$. Similarly, $s(b, a) \in I$ and so we conclude that $0 / \vartheta_{\mathrm{A}}(a, b) \subseteq I$.

For the converse, apply Proposition 2.8 to each $\mathrm{A} \in \mathscr{V}$, taking $I=(0)_{\mathrm{A}}$ and $\alpha=0_{\mathrm{A}}$. 
The second characterization of congruence quasi-orderable subtractive varieties is based on the characterization of congruence orderable varieties in [15]. If $\mathscr{V}$ is a subtractive variety, the class

$$
\mathscr{V}_{\varepsilon}=\left\{\mathbf{A} \in \mathscr{V}:(0)_{\mathbf{A}}^{\varepsilon}=0_{\mathbf{A}}\right\}
$$

has been investigated in [4] and it has also very strong connections with Blok and Pigozzi's theory of algebraizability [7]. In [4, Section 3] some of these connections are discussed (but see also [8] for additional information). If $\mathscr{V}$ is also congruence quasi-orderable, then via Corollary 2.2 and Lemma 2.4

$$
\begin{aligned}
\mathscr{V}_{\varepsilon} & =\left\{\mathbf{A} / \approx_{\mathbf{A}}: \mathbf{A} \in \mathscr{V}\right\} \\
& =\{\mathbf{A} \in \mathscr{V}: \text { for all } a, b \in A, s(a, b)=s(b, a)=0 \text { implies } a=b\},
\end{aligned}
$$

and therefore $\mathscr{V}_{\varepsilon}$ is a quasivariety, consisting precisely of the congruence orderable algebras in $\mathscr{V}$. This fact is an effective tool for showing that a subtractive variety is not congruence quasi-orderable.

EXAMPLE 2.10. Let $\mathbf{A}=\langle\{0, a, b, 1\}, s\rangle$ be the algebra whose $s$-table is

\begin{tabular}{c|cccc}
$s$ & 0 & $a$ & $b$ & 1 \\
\hline 0 & 0 & 0 & 0 & 0 \\
$a$ & $a$ & 0 & 1 & 0 \\
$b$ & $b$ & 0 & 0 & 1 \\
1 & 1 & 0 & 0 & 0
\end{tabular}

The variety $\mathscr{V}=\mathbb{V}(\mathbf{A})$ is subtractive (witness $s(x, y))$ and one easily sees that $\mathbf{A}$ is simple, hence $\mathbf{A} \in \mathscr{V}_{\varepsilon}$. However, $\mathbf{B}=\langle\{0, a, 1\}, s\rangle$ is a subalgebra of $\mathbf{A}$ and $0 / \vartheta_{\mathbf{B}}(a, 1)=\{0\}$. Hence $\mathbf{B} \notin \mathscr{V}_{\varepsilon}$, so $\mathscr{V}_{\varepsilon}$ is not closed under subalgebras and it is not a quasivariety. Thus $\mathscr{V}$ cannot be congruence quasi-orderable.

If $\mathscr{K}$ is a class of algebras and $\mathbf{A} \in \mathscr{K}$, then a $\mathscr{K}$-congruence of $\mathbf{A}$ (a relative congruence of $\mathbf{A}$ ) is a $\theta \in \operatorname{Con}(\mathbf{A})$ such that $\mathbf{A} / \theta \in \mathscr{X}$. If $\mathscr{X}$ is a quasivariety, then the relative congruences form an algebraic lattice $\operatorname{Con}_{\mathscr{X}}(\mathrm{A})$, in general not a sublattice of $\operatorname{Con}(\mathbf{A})$.

If $\mathscr{V}$ is a congruence quasi-orderable subtractive variety and $\mathrm{A} \in \mathscr{V}_{\varepsilon}$, we denote with $\mathrm{Con}_{\varepsilon}(\mathrm{A})$ the lattice of $\mathscr{V}_{\varepsilon}$-congruences of $\mathrm{A}$. It is easy to check that

$$
\operatorname{Con}_{\varepsilon}(\mathbf{A})=\left\{\theta \in \operatorname{Con}(\mathbf{A}):(0 / \theta)^{\varepsilon}=\theta\right\} .
$$

The following proposition summarizes the facts we are going to use from [4, Theorem 3.13 and Proposition 3.14]. 
PROPOSITION 2.11. Let $\mathscr{V}$ be a subtractive variety such that $\mathscr{V}_{\varepsilon}$ is a quasivariety.

(1) For each $\mathbf{A} \in \mathscr{V}$ and for any upward directed family $\left(I_{\gamma}: \gamma \in \Gamma\right)$ of ideals of A, we have

$$
\left(\bigcup_{\gamma \in \Gamma} I_{\gamma}\right)^{\varepsilon}=\bigcup_{\gamma \in \Gamma} I_{\gamma}^{\varepsilon} .
$$

(2) For each $\mathbf{A} \in \mathscr{V}_{\varepsilon}$, we have $\operatorname{Con}_{\varepsilon}(\mathbf{A}) \cong \mathrm{I}(\mathbf{A})$.

In particular, a congruence quasi-orderable subtractive variety satisfies (1) and (2) above.

We shall now characterize congruence quasi-orderable subtractive varieties. We start recalling that congruence orderable varieties (subtractive or not) have been characterized in [15] (but see also [1]). We recall that an algebra $\mathbf{A}$ is subdirectly irreducible if $\operatorname{Con}(\mathbf{A})$ has exactly one nontrivial minimal element $\mu$, called the monolith.

THEOREM 2.12. (1) If $\mathbf{A}$ is subdirectly irreducible and congruence orderable and $\mu$ is the monolith of $\mathbf{A}$, then $|0 / \mu|=2$ and all the other $\mu$-blocks are trivial.

(2) A pointed variety is congruence orderable if and only if each subdirectly irreducible member of $\mathscr{V}$ is congruence orderable.

A condition similar to (1) above must hold in any congruence quasi-orderable algebra.

LEMMA 2.13. Let $\mathbf{A}$ be a congruence quasi-orderable algebra. If $\mathbf{A}$ is subdirectly irreducible with monolith $\mu$, then either $0 / \mu=\{0\}$ or $|0 / \mu|=2$ and all the other $\mu$-blocks are trivial.

PROOF. Either $\approx_{A}=0_{A}$ or $\approx_{A}>0_{A}$. In the first case, $|0 / \mu|=2$, by Theorem 2.12. In the second one, $\mu \subseteq \approx_{A}$. Since $0 / \approx_{A}=\{0\}$ we get $0 / \mu=\{0\}$.

It is unlikely that the above condition is sufficient for a variety to be congruence quasi-orderable, not even in the subtractive case. The problem is that the class of congruence orderable algebras in a congruence quasi-orderable variety must be a quasivariety and the condition simply does not seem strong enough. We do not have any example yet, but to amend that we offer a different characterization.

THEOREM 2.14. For a subtractive variety $\mathscr{V}$ the following are equivalent.

(1) $\mathscr{V}$ is congruence quasi-orderable.

(2) $\mathscr{V}_{\varepsilon}$ is a quasivariety and in any relative subdirectly irreducible $\mathbf{A} \in \mathscr{V}_{\varepsilon}[$ that is, $\operatorname{Con}_{\varepsilon}(\mathrm{A})$ has a unique nontrivial minimal element] with relative monolith $\mu,|0 / \mu|=2$ and all the other blocks are trivial. 
PROOF. If $\mathscr{V}$ is congruence quasi-orderable, then $\mathscr{V}_{\varepsilon}$ is a quasivariety. If $\mathrm{A} \in \mathscr{V}_{\varepsilon}$, then $\mathbf{A}$ is congruence orderable and, by Proposition $2.11(2), \operatorname{Con}_{\varepsilon}(\mathbf{A}) \cong \mathrm{I}(\mathbf{A})$. Hence if $\mathbf{A}$ is relatively subdirectly irreducible, then $\mathbf{I}(\mathbf{A})$ has a unique minimal nontrivial ideal $I$, that must be principal, say $I=(d)_{\mathrm{A}}$. If $a, b \in A$ with $a, b \neq 0$, then $(d)_{\mathbf{A}} \subseteq(a)_{\mathbf{A}} \cap(b)_{\mathbf{A}}$. Now let $\mu$ be the relative monolith and suppose that $(a, b) \in \mu$. Since $\mathscr{V}$ is subtractive, $(d)=0 / \mu$ and for any $s(x, y)$ witnessing subtractivity for $\mathscr{V}$ we have

$$
s(a, b), s(b, a) \in 0 / \mu=(d)_{\mathrm{A}} \subseteq(a)_{\mathrm{A}} \cap(b)_{\mathrm{A}} .
$$

By (the proof of) Lemma 2.4, we get $a \approx_{\mathrm{A}} b$ and hence $a=b$, since A is congruence orderable. It follows that (2) holds.

Assume now (2) and suppose that $\mathscr{V}$ is not congruence quasi-orderable. Thus there exists an algebra $\mathrm{A} \in \mathscr{V}$ and $a, b \in A$ with $a \approx_{\mathrm{A}} b$, but $(a, b) \notin(0)_{\mathrm{A}}^{\varepsilon}$. Since we are in a subtractive variety in fact $(a)_{\mathbf{A}}=(b)_{\mathbf{A}}$ and hence $\left(a /(0)_{\mathbf{A}}^{\varepsilon}\right)_{\mathbf{A} /(0)_{A}^{\varepsilon}}=\left(b /(0)_{A}^{\varepsilon}\right)_{\mathbf{A} /(0))_{A}^{\varepsilon}}$. Therefore we may assume that there is an algebra $\mathrm{A} \in \mathscr{V}_{\varepsilon}$ and $a, b \in A$ with $a \approx_{\mathrm{A}} b$ but $a \neq b$. Then

Let now

$$
\vartheta_{\mathbf{A}}(a, b) \subseteq \vartheta_{\mathbf{A}}(0, a) \vee \vartheta_{\mathbf{A}}(0, b)=\vartheta_{\mathbf{A}}(0, a)
$$

$$
\mathscr{F}=\left\{\theta \in \operatorname{Con}_{\varepsilon}(\mathbf{A}):(a, b) \notin \theta\right\} .
$$

The set $\mathscr{F}$ is nonempty, since $0_{\mathrm{A}} \in \mathscr{F}$. Let now $\left(\theta_{\gamma}: \gamma \in \Gamma\right)$ be a chain in $\mathscr{F}$. Then $\theta=\bigvee_{\gamma \in \Gamma} \theta_{\gamma} \in \operatorname{Con}(\mathbf{A})$ and clearly $(a, b) \notin \theta$. Moreover, by Proposition 2.11 (1) and subtractivity,

$$
(0 / \theta)^{\varepsilon}=\left(0 / \bigvee_{\gamma \in \Gamma} \theta_{\gamma}\right)^{\varepsilon}=\left(\bigvee_{\gamma \in \Gamma} 0 / \theta_{\gamma}\right)^{\varepsilon}=\bigvee_{\gamma \in \Gamma}\left(0 / \theta_{\gamma}\right)^{\varepsilon}=\bigvee_{\gamma \in \Gamma} \theta_{\gamma}=\theta,
$$

hence $\theta \in \mathscr{F}$. By Zorn's Lemma, $\mathscr{F}$ has a maximal element $\beta$ and, by the second homomorphism theorem, $\mathbf{A} / \boldsymbol{\beta}$ is a relative subdirectly irreducible algebra in $\operatorname{Con}(\mathbf{A})$. Moreover, the pair $(a / \beta, b / \beta)$ must lie in the relative monolith of $\mathbf{A} / \beta$. By hypothesis $0 / \beta \in\{a / \beta, b / \beta\}$, say $0 / \beta=a / \beta$. But then

$$
\vartheta_{\mathbf{A}}(a, b) \subseteq \vartheta_{\mathbf{A}}(0, a) \subseteq \beta,
$$

a contradiction. Thus (2) implies (1).

REMARK 2.15. (1) It is easy enough to find a subtractive variety $\mathscr{V}$ such that $\mathscr{V}_{\varepsilon}$ is a proper quasivariety. There exists a variety $\mathscr{V}$ ([4, Example 4.5]) with the following properties:

- $\mathscr{V}$ has basic operations $*, 0$, where $*$ is binary and 0 is constant. 
- $\mathscr{V}$ is subtractive and for any $\mathbf{A} \in \mathscr{V}$ the relation $\{(a, b): a * b=b * a=0\}$ is a congruence of $\mathbf{A}$, thus it coincides with $(0)_{\mathbf{A}}^{\epsilon}$.

- $\mathscr{Y}_{\varepsilon}$ is termwise equivalent to the class of BCK-algebras, a quasivariety that is not a variety [21].

It turns out that $\mathscr{V}$ is exactly the variety generated by the quasivariety of BCK-algebras. However $\mathscr{V}$ is non congruence quasi-orderable.

(2) In the sequel we shall see that $\mathscr{V}_{\varepsilon}$ is a variety when $\mathscr{V}$ is subtractive, congruence quasi-orderable and has other properties (Corollary 3.3, Theorem 4.2 and Corollary 4.12). In fact, all the examples we know of subtractive congruence quasiorderable varieties satisfy the hypotheses of the theorem and corollaries above, so we do not know what happen in the general case.

(3) A question related to the problem in (2) is the following: let $\mathscr{Q}$ be a proper quasivariety of subtractive congruence orderable algebras. Is $\mathbb{V}(\mathscr{Q})$ congruence quasiorderable? In particular, it seems crucial to determine necessary and sufficient conditions on $\mathbf{A}$ to conclude that $\mathbf{A} / \alpha$ is congruence quasi-orderable for any $\alpha \in \operatorname{Con}(\mathbf{A})$. The condition in Proposition 2.8 is sufficient but certainly not necessary.

\section{Quasi-permutability of congruences}

An equivalential algebra [16] is an algebra $\langle A, \oplus, 0\rangle$ satisfying the following identities

(E1) $(x \oplus x) \oplus y \approx y$;

(E2) $((x \oplus y) \oplus z) \oplus z \approx(x \oplus z) \oplus(y \oplus z)$;

(E3) $((x \oplus y) \oplus((x \oplus z) \oplus z)) \oplus((x \oplus z) \oplus z) \approx x \oplus y$.

It can be shown that $x \oplus x \approx y \oplus y$ is an identity of equivalential algebras. Hence $0 \approx x \oplus x$ is an equationally definable constant satisfying also $x \oplus 0 \approx x \approx 0 \oplus x$. Moreover $x \oplus y \approx 0$ implies $x \approx y$. The variety of equivalential algebras is ideal determined, it is not congruence distributive (since it contains the variety of Boolean groups) and it is congruence permutable with the Mal'cev term

$$
m(x, y, z)=((x \oplus y) \oplus z) \oplus(((x \oplus z) \oplus z) \oplus x) .
$$

Let $S=\langle S, \vee, 0\rangle$ be a lower bounded semilattice. It may happen, for some $a, b \in S$, that the set $\{c: a \vee c=b \vee c\}$ has a smallest element. In this case it is denoted by $a \oplus b$ and is called the dual symmetric relative pseudocomplement of $a$ and $b$. The lemma below is well known and it is just a consequence of the definition of equivalential algebras. 
LEMMA 3.1. If a subset $E$ of a lower bounded join semilattice contains 0 and each two elements in $E$ have a dual symmetric relative pseudocomplement that belongs to $E$, then $\langle E, \oplus, 0\rangle$ is an equivalential algebra.

For any pointed algebra $\mathbf{A}$ the compact ideals of $\mathbf{A}$ form a lower bounded join semilattice, denoted by $\mathrm{CI}(\mathbf{A})$. If $\mathbf{A}$ is also subtractive and congruence quasi-orderable, then for any $a, b \in A$ the ideal $0 / \vartheta_{\mathbf{A}}(a, b)$ is compact (Corollary 2.6) and moreover $0 / \vartheta_{\mathrm{A}}(a, b)=(a)_{\mathrm{A}} \oplus(b)_{\mathrm{A}}$, the dual symmetric relative pseudocomplement of $(a)_{\mathrm{A}}$ and $(b)_{\mathbf{A}}$ in $\operatorname{CI}(\mathbf{A})$ (Theorem 2.9). We will investigate the case in which $\left.0 / \vartheta_{(} a, b\right)_{\mathbf{A}}$ is also principal; from now on we denote by $\operatorname{PI}(\mathbf{A})$ the set of principal ideals of a pointed algebra $\mathbf{A}$.

Let $\mathbf{A}$ be a subtractive algebra. An equivalential term of $\mathbf{A}$ is a binary term $e(x, y)$ such that $0 / \vartheta_{\mathrm{A}}(a, b)=(e(a, b))_{\mathrm{A}}$ for all $a, b \in A$.

THEOREM 3.2. For a congruence quasi-orderable subtractive variety $\mathscr{V}$ the following are equivalent:

(1) $\mathscr{V}$ has an equivalential term.

(2) For all $\mathbf{A} \in \mathscr{V}$ and any $a, b \in A, 0 / \vartheta_{\mathbf{A}}(a, b)$ is principal.

(3) There is a binary term e such that $\langle A, e, 0\rangle / \approx_{\mathrm{A}}$ is an equivalential algebra for all $\mathbf{A} \in \mathscr{V}$.

(4) There is a binary term $e$ such that for any $\mathbf{A} \in \mathscr{V}$ and $a \in A, e(a, a)=0$, $(e(a, 0))_{\mathbf{A}}=(a)_{\mathbf{A}}=(e(0, a))_{\mathbf{A}}$.

(5) There is a binary term $e$ such that for any $\mathbf{A} \in \mathscr{V}$ and $a, b \in A$,

$$
(a)_{\mathrm{A}}=(b)_{\mathrm{A}} \text { if and only if } e(a, b)=0 .
$$

Proof. (1) implies (2) from the definition of equivalential term. Assume then (2) and let $\mathbf{F}$ be the algebra in $\mathscr{V}$ freely generated by $\left\{x, y, z_{1}, z_{2}, \ldots\right\}$. Then $0 / \vartheta_{\mathbf{F}}(x, y)$ is principal, hence there is a term $t\left(x, y, z_{1}, \ldots, z_{n}\right)$ such that $0 / \vartheta_{\mathbf{F}}(x, y)=$ $\left(t\left(x, y, z_{1}, \ldots, z_{n}\right)\right)_{\mathrm{F}}$. Let $e(x, y)=t(x, y, x, \ldots, x)$. We show that $e(x, y)$ is an equivalential term for $\mathscr{V}$ by proving that for any $\mathbf{A} \in \mathscr{V}$ and $a, b, c \in A$

$$
c \in 0 / \vartheta_{\mathbf{A}}(a, b) \text { if and only if } c \in(e(a, b))_{\mathbf{A}} .
$$

Let $\mathbf{A}$ be a finitely generated algebra in $\mathscr{V}$. Let $\varphi$ be an onto homomorphism from $\mathbf{F}$ to A such that $\varphi(x)=\varphi\left(z_{1}\right)=\cdots=\varphi\left(z_{n}\right)=a$ and $f(y)=b$. Let $c \in A$ and lett $u \in F$ with $\varphi(u)=c$. Using Corollary 2.6 and Proposition 2.1 we get the following chain of equivalences.

$$
\begin{array}{lll}
c \in 0 / \vartheta_{\mathbf{A}}(a, b) & \text { if and only if } & c \in\langle s(a, b), s(b, a)\rangle_{\mathbf{A}} \\
& \text { if and only if } & \varphi(u) \in\langle s(\varphi(x), \varphi(y)), s(\varphi(y), \varphi(x))\rangle_{\mathbf{A}}
\end{array}
$$




$$
\begin{array}{ll}
\text { if and only if } & u \in\langle s(x, y), s(y, x)\rangle_{\mathrm{F}} \vee 0 / \operatorname{ker}(\varphi) \\
\text { if and only if } & u \in 0 / \vartheta_{\mathrm{F}}(x, y) \vee 0 / \operatorname{ker}(\varphi) \\
\text { if and only if } & u \in\left(t\left(x, y, z_{1}, \ldots, z_{n}\right)\right)_{\mathrm{F}} \vee 0 / \operatorname{ker}(\varphi) \\
\text { if and only if } & \varphi(u) \in\left(\varphi\left(t\left(x, y, z_{1}, \ldots, z_{n}\right)\right)\right)_{\mathrm{A}} \\
\text { if and only if } & c \in(t(a, b, a, \ldots, a))_{\mathrm{A}}=(e(a, b))_{\mathrm{A}} .
\end{array}
$$

Hence (3.1) holds for finitely generated algebras. Suppose that $\mathbf{B}$ is not finitely generated and let $a, b, c \in A$ with $c \in 0 / \vartheta_{\mathbf{B}}(a, b)$. Since $0 / \vartheta_{\mathbf{B}}(a, b)$ is compact, there exists a finitely generated subalgebra $\mathbf{A}$ of $\mathbf{B}$ with $c \in 0 / \vartheta_{\mathbf{A}}(a, b)$. Therefore, $c \in(e(a, b))_{\mathbf{A}} \subseteq(e(a, b))_{\mathbf{B}}$. If $c \in(e(a, b))_{\mathbf{B}}$, since $(e(a, b))_{\mathbf{B}}$ is principal, there exists a finitely generated subalgebra $\mathbf{A}$ of $\mathbf{B}$ with $c \in(e(a, b))_{\mathbf{A}}$. Therefore, $c \in$ $0 / \vartheta_{\mathbf{A}}(a, b) \subseteq 0 / \vartheta_{\mathbf{B}}(a, b)$. We have shown that (1) and (2) are equivalent.

If (2) holds, then $\left\langle\mathrm{PI}(\mathrm{A}), \oplus,(0)_{\boldsymbol{A}}\right\rangle$ is an equivalential algebra by Theorem 2.9 and Lemma 3.1. The mapping $a \mapsto(a)_{A}$ is a homomorphism from $\langle A, e, 0\rangle$ to $\langle\mathrm{PI}(\mathbf{A}), \oplus, 0\rangle$ whose kernel is $\approx_{A}$. Therefore, (3) holds. If (3) holds, then (4) holds since the equations $e(x, x) \approx 0$ and $e(x, 0) \approx x \approx e(0, x)$ hold in the equivalential algebra $\langle A, e, 0\rangle / \approx_{\mathrm{A}}$.

Let us assume (4) First note that $e(a, b) \vartheta_{\mathrm{A}}(a, b) e(a, a)=0$, hence $(e(a, b))_{\mathrm{A}} \subseteq$ $0 / \vartheta_{\mathrm{A}}(a, b)$. Next

$$
e(0, b) \vartheta_{\mathrm{A}}(0, a) e(a, b) \vartheta_{\mathrm{A}}(0, e(a, b)) 0
$$

hence $e(0, b) \in(a)_{\mathrm{A}} \vee(e(a, b))_{\mathrm{A}}$. Since the latter is an ideal, from the hypothesis we have $b \in(a)_{\mathbf{A}} \vee(e(a, b))_{\mathbf{A}}$. A symmetrical argument yields $a \in(b)_{\mathbf{A}} \vee(e(a, b))_{\mathbf{A}}$, so

$$
(a)_{\mathrm{A}} \vee(e(a, b))_{\mathrm{A}}=(b)_{\mathrm{A}} \vee(e(a, b))_{\mathrm{A}}
$$

By Theorem 2.9, $0 / \vartheta_{\mathrm{A}}(a, b) \subseteq(e(a, b))_{\mathrm{A}}$ and therefore equality holds. Thus (4) implies (2).

Clearly (3) implies (5) via the properties of equivalential algebras. Finally assume (5) and consider the operation on $\mathrm{CI}(\mathbf{A})$ defined by $(a)_{\mathbf{A}} \oplus(b)_{\mathbf{A}}=(e(a, b))_{\mathbf{A}}$. We claim that $e(x, y)$ is the symmetric dual relative pseudocomplement of $a$ and $b$ in $\mathrm{CI}(\mathbf{A})$, that is, $(e(a, b))_{\mathbf{A}}=0 / \vartheta_{\mathbf{A}}(a, b)$. By hypothesis $e(a, a)=0$, hence

$$
e(a, b) \vartheta_{\mathrm{A}}(a, b) e(a, a)=0
$$

so that $(e(a, b))_{\mathbf{A}} \subseteq 0 / \vartheta_{\mathbf{A}}(a, b)$. Let now $(e(a, b))_{\mathbf{A}}=0 / \theta$ for some $\theta \in \operatorname{Con}(\mathbf{A})$. Then $e(a / \theta, b / \theta)=0 / \theta$, which implies that $(a / \theta)_{\mathbf{A} / \theta}=(b / \theta)_{\mathbf{A} / \theta}$. An application of Corollary 2.2 gives

$$
(a)_{\mathrm{A}} \vee(e(a, b))_{\mathrm{A}}=(b)_{\mathrm{A}} \vee(e(a, b))_{\mathrm{A}}
$$

By Theorem 2.9, $0 / \vartheta_{\mathrm{A}}(a, b) \subseteq(e(a, b))_{\mathrm{A}}$, therefore equality holds. Thus (5) implies (2). 
COROLLARY 3.3. Let $\mathscr{V}$ be a subtractive congruence quasi-orderable variety with an equivalential term. Then the class $\mathscr{V}_{\varepsilon}$ is a congruence orderable congruence permutable variety.

PROOF. It suffices to show that the class $\mathscr{V}_{\varepsilon}$ is closed under homomorphic images. In fact $\mathscr{V}_{\varepsilon}=\{\mathrm{A} \in \mathscr{V}:\langle A, e, 0\rangle$ is an equivalential algebra $\}$, thus, if it is a variety then the Mal'cev term for equivalential algebras works as a Mal'cev term for $\mathscr{V}_{\varepsilon}$. If $\mathrm{A} \in \mathscr{V}_{\varepsilon}$ and $\mathbf{B}$ is a homomorphic image of $\mathbf{A}$, then $\langle B, e, 0\rangle$ is clearly again an equivalential algebra. Thus $\mathrm{B} \in \mathscr{V}_{\varepsilon}$ and $\mathscr{V}_{\varepsilon}$ is a variety.

In [15] it is basically shown that congruence orderable varieties with an equivalential term coincide with congruence permutable congruence orderable varieties. We shall see that similar result holds for congruence quasi-orderable varieties.

Let $\mathbf{A}$ be a pointed algebra. A quasi-Mal'cev term for $\mathbf{A}$ is a ternary term $w(x, y, z)$ such that for any $a, b \in A$

$$
(w(a, b, b))_{\mathrm{A}}=(a)_{\mathrm{A}} \quad(w(a, a, b))_{\mathrm{A}}=(b)_{\mathrm{A}} .
$$

The following proposition is essentially contained in [3, (1.12)]. We reproduce the easy proof for the reader.

PROPOSITION 3.4. If $\mathscr{V}$ is a pointed variety with a quasi-Mal'cev term $w(x, y, z)$, then $\mathscr{V}$ is subtractive.

Proof. Let $t(x, y)=w(x, y, 0)$. If $\mathbf{A} \in \mathscr{V}$, then $(t(a, 0))_{\mathbf{A}}=(a)_{\mathbf{A}}$ and $(t(a, a))=$ $(0)_{\mathbf{A}}$, that is, $t(a, a)=0$. Now suppose that for some $\varphi, \theta \in \operatorname{Con}(\mathbf{A})(a, 0) \in \theta \circ \varphi$. Then there is a $b \in A$ with $a \theta b \varphi 0$ and so $t(a, a)=0 \theta t(a, b) \varphi t(a, 0)$. It follows that $t(a, 0) \in 0 /(\theta \circ \varphi)$ and the latter is an ideal, since $\theta \circ \varphi$ is clearly a reflexive subalgebra of $\mathbf{A} \times \mathbf{A}$. From $(t(a, 0))_{\mathbf{A}}=(a)_{\mathbf{A}}$ we deduce that $a \in 0 /(\theta \circ \varphi)$ as well, and so the congruences of $\mathbf{A}$ permute at 0 .

From Lemma 2.13 it follows that the monolith $\mu$ any congruence orderable subdirectly irreducible $\mathbf{A}$ has a unique nontrivial block, that is $0 / \mu$, and that block contains only one element different from 0 . Following [15] we shall denote this element by $\star_{A}$. The following lemma is crucial.

LEMMA 3.5. Suppose that $\mathscr{V}$ is a congruence quasi-orderable subtractive variety and let $t(x, y)$ be a binary term such that for any congruence orderable subdirectly irreducible $\mathbf{A} \in \mathscr{V}, t\left(0, \star_{\mathbf{A}}\right)=\star_{\mathbf{A}}$. If $t(x, x) \approx 0$ holds in $\mathscr{V}$, then for any algebra $\mathbf{B} \in \mathscr{V}$ and $b \in B$ we have $t(0, b) \approx_{\mathrm{B}} b$. 
PROOF. It is clear that for any $b \in B$ we have $(t(0, b))_{\mathbf{B}} \subseteq(b)_{\mathrm{B}}$, since $t(b, b)=0$. Suppose that the inclusion is proper, that is, $(0, b) \notin \vartheta_{\mathbf{B}}(0, t(0, b))$. Pick a maximal congruence $\beta$ containing $(0, t(0, b))$ but not $(0, b)$. Then of course $\mathrm{B} / \beta$ is subdirectly irreducible; by maximality of $\beta$, the monolith of $\mathbf{B} / \beta$ must contain the pair $(0 / \beta, b / \beta)$, which are distinct, since $(0, b) \notin \beta$. From Lemma 2.13 follows that $\mathbf{B} / \beta$ is congruence orderable and $\star_{\mathrm{B} / \beta}=b / \beta$. Hence

$$
b / \beta=t(0 / \beta, b / \beta)=t(0, b) / \beta=0 / \beta
$$

a contradiction. This yields the conclusion.

THEOREM 3.6. For a congruence quasi-orderable variety $\mathscr{V}$ the following are equivalent:

(1) $\mathscr{V}$ is subtractive and has an equivalential term.

(2) $\mathscr{V}$ has a quasi-Mal'cev term.

PROOF. If $\mathscr{V}$ is subtractive and has an equivalential term, then by Theorem 3.2 there is a binary term $e(x, y)$ such that for any $\mathbf{A} \in \mathscr{V},\langle A, e, 0\rangle / \approx_{\mathbf{A}}$ is an equivalential algebra. But equivalential algebras are congruence permutable, hence the Mal'cev term for equivalential algebras works as a quasi-Mal'cev term for $\mathscr{V}$.

Conversely, if $\mathscr{V}$ has a quasi-Mal'cev term $w(x, y, z)$ then it is subtractive by Proposition 3.4. Define a binary term

$$
e(x, y)=w(w(0, w(0, x, \bar{y}), w(0, y, x)), 0, w(0, x, y)) .
$$

Then, using the trivial fact that $w(0, x, x)=0$ we get that $e(x, x)=0$ holds in $\mathscr{V}$.

Now let $\mathbf{A}$ be a congruence orderable subdirectly irreducible member of $\mathscr{V}$. Then from $\left(w\left(\star_{\mathbf{A}}, 0,0\right)\right)_{\mathbf{A}}=\left(\star_{\mathbf{A}}\right)_{\mathbf{A}}=\left\{0, \star_{\mathbf{A}}\right\}$ we get $w\left(\star_{\mathbf{A}}, 0,0\right)=\star_{\mathbf{A}}$ and similarly $w\left(0,0, \star_{\mathbf{A}}\right)=\star_{\mathbf{A}}$. So

$$
\begin{aligned}
& \boldsymbol{e}\left(0, \star_{\mathbf{A}}\right)=w\left(w\left(0, \star_{\mathbf{A}}, w\left(0, \star_{\mathbf{A}}, 0\right)\right), 0, \star_{\mathbf{A}}\right), \\
& \boldsymbol{e}\left(\star_{\mathbf{A}}, 0\right)=w\left(w\left(0, w\left(0, \star_{\mathbf{A}}, 0\right), \star_{\mathbf{A}}\right), \mathbf{0}, w\left(0, \star_{\mathbf{A}}, 0\right)\right) .
\end{aligned}
$$

Now $w\left(0, \star_{\mathbf{A}}, 0\right) \in\left\{0, \star_{\mathbf{A}}\right\}$ and considering either one of the possible values we get $e\left(0, \star_{A}\right)=e\left(\star_{A}, 0\right)=\star_{A}$ in both cases. Hence by Lemma 3.5, for any $\mathbf{B} \in \mathscr{V}$ and $b \in B,(e(b, 0))_{\mathrm{B}}=(b)_{\mathrm{B}}=(e(0, b))_{\mathrm{B}}$. An application of Theorem 3.2 gives the desired conclusion.

REMARK 3.7. We could add a third equivalent condition to Theorem 3.6. In fact congruence quasi-orderable varieties with an equivalential term turn out to coincide with congruence quasi-orderable varieties that are $d$-subtractive in the sense of [4]. Though this connection seems worth investigating further, it would have led us away from the path we are following in this paper. 


\section{Equationally definable principal ideals}

A subtractive variety $\mathscr{V}$ has equationally definable principal ideals (EDPI for short) if there exists a binary term $p(x, y)$ such that for any $\mathbf{A} \in \mathcal{V}$ and $a, b \in A$

$$
a \in(b)_{\mathrm{A}} \quad \text { if and only if } \quad p(a, b)=0 .
$$

Such varieties have been studied mainly in $[3,5]$ and congruence orderable varieties with EDPI have been characterized in [1]. That characterization extends easily to congruence quasi-orderable subtractive varieties. The fact about subtractive varieties with EDPI that we shall use are contained in the following.

THEOREM 4.1. (1) ([5, Theorem 3.4]) If a subtractive variety has EDPI, then there is a binary term $x * y$ witnessing both subtractivity and EDPI.

(2) ([3, Proposition 4.2]) $\mathscr{V}$ is a subtractive EDPI variety if and only if for any algebra $\mathbf{A} \in \mathscr{V}$ the join semilattice $\mathrm{CI}(\mathbf{A})$ of compact ideals is a dual Brouwerian semilattice.

(3) ([5, Proposition 3.2]) A subtractive variety with EDPI is ideal distributive, that is, the ideal lattices of its members are distributive.

(4) ([5, Theorem 3.1]) An ideal-determined variety has EDPI if and only if it has equationally definable principal congruences.

We recall that a dual Brouwerian semilattice is a join semilattice with dual relative pseudocomplementation, that is, an algebra $\langle A, *, \vee, 0\rangle$ such that

$$
a * b \leq c \text { if and only if } \quad b \leq a \vee c
$$

A dual Hilbert algebra is a *-subreduct of a dual Brouwerian semilattice.

THEOREM 4.2. Let $\mathscr{V}$ be a congruence quasi-orderable subtractive variety with EDPI. Then $\mathcal{V}_{\varepsilon}$ is a congruence orderable subtractive variety with equationally definable principal congruences.

PROOF. Once we show that $\mathscr{V}_{\varepsilon}$ is closed under homomorphic images, the rest follows from our previous results. Let $\mathbf{A} \in \mathscr{V}$. We can repeat the argument of [1, Theorem 3.4] to conclude that the mapping $a \mapsto(a)_{\mathrm{A}}$ is a homomorphism from $\langle A, *, 0\rangle$ onto $\operatorname{PI}(\mathbf{A})$, whose kernel is $\approx_{\mathbf{A}}$. Hence, $\langle A, *, 0\rangle / \approx_{\mathbf{A}}$ is a dual Hilbert algebra (since it is the reduct of a dual Brouwerian semilattice). Now $\mathbf{A} \in \mathscr{V}_{\varepsilon}$ if and only if $\approx_{\mathbf{A}}=0_{\mathrm{A}}$, thus if and only if $\langle A, *, 0\rangle$ is a dual Hilbert algebra. Let $\mathbf{A} \in \mathscr{V}$ and let $f: \mathbf{A} \rightarrow \mathbf{B}$ a onto homomorphism. Since $*$ is a term of $\mathscr{V}$, then $f$ must respect $*$. Hence any equation involving $*$ holds in $\mathbf{B}$ as well. Therefore, $\langle B, *, 0\rangle$ is a Hilbert algebra and $\mathrm{B} \in \mathscr{V}_{\varepsilon}$. 
If $\mathbf{A}$ belongs to a congruence quasi-orderable variety $\mathscr{V}$, then it is possible to describe the structure of $\langle A, *, 0\rangle$ even if $\mathbf{A} \notin \mathscr{V}_{\varepsilon}$ and the following easy lemma (that appears already in [1]) is the key.

LEMMA 4.3. Let $\mathscr{V}$ be a subtractive variety with EDPI. There exists a binary term $x * y$ of $\mathscr{V}$ such that, for each $\mathrm{A} \in \mathscr{V}, I \in \mathrm{I}(\mathrm{A})$ and $a, b \in A$

$$
a * b \in I \quad \text { if and only if } \quad b \in(a)_{\mathrm{A}} \vee I .
$$

PROOF. By Theorem 4.1(1) there is a binary term $x * y$ witnessing both subtractivity and EDPI and by Theorem 4.1(2) for any $\mathbf{A} \in \mathscr{V}$ the semilattice $\mathrm{CI}(\mathbf{A})$ of compact ideals is a dual Brouwerian semilattice. We denote again by $*$ the dual relative pseudocomplementation in $\mathrm{CI}(\mathbf{A})$. Let $a * b \in I, I=0 / \theta$ for some $\theta \in \operatorname{Con}(\mathbf{A})$. Thus, $a / \theta * \beta / \theta=0 / \theta$ and so $b / \theta \in(a / \theta)_{\mathrm{A} / \theta}$. Hence there is an ideal term $t(\vec{x}, y)$ in $y$ such that $b / \theta=t(\vec{u} / \theta, a / \theta)$ for some $\vec{u} \in A$. This in turn implies

$$
b \theta t(\vec{u}, a) \vartheta_{\mathrm{A}}(0, a) t(\vec{u}, 0)=0
$$

and so

$$
b \in 0 /\left(\theta \circ \vartheta_{\mathrm{A}}(0, a)\right)=0 / \vartheta_{\mathrm{A}}(0, a) \vee 0 / \theta=(a)_{\mathrm{A}} \vee I,
$$

where we have used the fact that in subtractive varieties congruences permute at 0 . The converse implication is proved similarly.

Let $\mathscr{M}$ be the variety of pointed binars $\langle A, *, 0\rangle$ axiomatized by:

(M1) $x * 0 \approx 0$.

(M2) $0 * x \approx x$.

(M3) $(x *(y * z)) *((x * y) *(x * z)) \approx 0$.

(M4) $(x * y) *((y * z) *(x * z)) \approx 0$.

Note that (M2), (M3) and (M4) imply $x * x \approx 0$, so $\mathscr{M}$ is subtractive. Such algebras have been introduced in [5, Example 3.7] and they are called MINI algebras. It is also shown that for $\mathbf{A} \in \mathscr{M}$ and $a, b \in A$

$$
b \in(a)_{\mathrm{A}} \text { if and only if } a * b=0,
$$

thus $\mathscr{M}$ has EDPI.

PROPOSITION 4.4. $\mathscr{M}$ is congruence quasi-orderable but not congruence orderable.

ProOF. We must show that $\approx_{\mathbf{A}} \in \operatorname{Con}(\mathbf{A})$ for each $\mathbf{A} \in \mathscr{M}$. Since $\mathscr{M}$ has EDPI with witness term $x * y$

$$
\approx_{\mathrm{A}}=\{(a, b): a * b=b * a=0\} .
$$


Since $\approx_{\mathrm{A}}$ is always an equivalence, we have to show that, for any $a, b, c \in A, a \approx_{\mathrm{A}} b$ implies both $a * c \approx_{\mathrm{A}} b * c$ and $c * a \approx_{\mathrm{A}} c * b$. The first statement follows from (M1), (M2) and (M4) and the second from (M1), (M2) and (M3).

To show that $\mathscr{M}$ is not congruence orderable we argue by contradiction. If $\mathscr{M}$ were congruence orderable, then it would be ideal-determined ([1, Theorem 2.1]) and hence it would have equationally definable principal congruences (Theorem 4.1). It is well-known that any variety with that property must be congruence distributive [17]. However $\mathscr{M}$ is not even congruence modular. Consider the algebra $\mathbf{A}=$ $\langle\{0,1,2,3,4,5\}, *, 0\rangle$, where

$$
x * y= \begin{cases}y & \text { if } x=0 \\ 0 & \text { otherwise }\end{cases}
$$

It is easy to see that $\mathbf{A} \in \mathscr{M}$ and that a proper congruence of $\mathbf{A}$ is simply a partition to which $\{0\}$ belongs. Now it is easily seen that Con(A) is a nonmodular lattice.

Let $\mathbf{A}$ be a MINI algebra. An $n$-ary operation $f$ on $A$ is compatible if for any $i \leq n$ and any $a, b \in A$

$$
(a * b) *\left((b * a) *\left(\tilde{f_{i}}(a) * \tilde{f_{i}}(b)\right)\right)=0,
$$

where $\tilde{f_{i}}(x)=f\left(z_{1}, \ldots, z_{i-1}, x, z_{i+1}, \ldots, z_{n}\right)$. It can be checked that the compatible operations of $\mathbf{A}$ are exactly those that preserve each MINI algebra congruence.

THEOREM 4.5. A pointed variety is congruence quasi-orderable, subtractive and has EDPI if and only if it is termwise equivalent to a variety of MINI algebras with compatible operations.

PROOF. In a MINI algebra with compatible operations the congruences (and hence the ideals) depend only on the MINI algebra operation. Therefore any such variety is congruence quasi-orderable, subtractive and has EDPI.

Let $\mathscr{V}$ be congruence quasi-orderable, subtractive and with EDPI. By Theorem 4.1 (1) there is a binary term $x * y$ of $\mathscr{V}$ such that (M1) and (M2) hold in $\mathscr{V}$ and moreover $\langle A, *, 0\rangle / \approx_{\mathrm{A}}$ is a dual Hilbert algebra for any $\mathbf{A} \in \mathscr{V}$. Now (M3) is an equation in the language $\{*, 0\}$ that holds in dual Hilbert algebras. So for any $\mathbf{A} \in \mathcal{V}$ and $a, b, c \in A$

$$
\left(a / \approx_{\mathbf{A}} *\left(b / \approx_{\mathbf{A}} * c / \approx_{\mathbf{A}}\right)\right) *\left(\left(a / \approx_{\mathbf{A}} * b / \approx_{\mathbf{A}}\right) *\left(a / \approx_{\mathbf{A}} * c / \approx_{\mathbf{A}}\right)\right)=0 / \approx_{\mathbf{A}} .
$$

Clearly, $0 / \approx_{A}=\{0\}$ and therefore

$$
(a *(b * c)) *((a * b) *(a * c))=0
$$


in A. It follows that (M3) holds in $\mathscr{V}$. The proof that (M4) holds in $\mathscr{V}$ is similar, hence $\langle A, *, 0\rangle$ is a MINI algebra for each $\mathrm{A} \in \mathcal{V}$.

To conclude the proof we must show that any basic operation of $\mathbf{A}$ is compatible in the above sense. Let $f(x)$ be a basic operation, which can be taken to be unary without loss of generality. Let $a, b \in A$ and let

$$
(a * b)_{\mathrm{A}} \vee(b * a)_{\mathrm{A}}=0 / \theta
$$

for some $\theta \in \operatorname{Con}(\mathbf{A})$. Then of course $a / \theta * b / \theta=b / \theta * a / \theta=0 / \theta$ and so $(a / \theta)_{\mathbf{A} / \theta}=(b / \theta)_{\mathbf{A} / \theta}$, implying $a / \theta \approx_{\mathbf{A} / \theta} b / \theta$. Therefore, $f(a) / \theta \approx_{\mathbf{A} / \theta} f(b) / \theta$ and hence $(f(a) * f(b)) / \theta=0 / \theta$ implying

$$
f(a) * f(b) \in 0 / \theta=(a * b)_{\mathbf{A}} \vee(b * a)_{\mathbf{A}} .
$$

Now we apply twice Lemma 4.3 to get

$$
(a * b) *((b * a) *(f(a) * f(b)))=0
$$

and hence $f$ is compatible.

REMARK 4.6. At this point the reader might wonder why we did not give a characterization similar to the one in Theorem 4.5 for subtractive congruence quasi-orderable varieties with an equivalential term. The reason is that such characterization, if any, must be very difficult to formulate. We can show easily enough that if $\mathscr{V}$ is a subtractive congruence quasi-orderable variety with an equivalential term $e(x, y)$ and $\mathrm{A} \in \mathscr{V}$, then the operations of $\mathbf{A}$ do satisfy a weak form of compatibility; in fact if $f$ is a (unary) operation of $\mathbf{A}$, then for each $a, b \in A$

$$
e(f(a), f(b)) \in(e(a, b))_{\mathbf{A}} .
$$

However it seems difficult to describe this property in terms of equations. The main difference between subtractive congruence quasi-orderable varieties with an equivalential term and subtractive congruence quasi-orderable varieties with EDPI seems to be the following. In the first case (Theorem 3.2) the relation $\approx_{A}$ is termdefinable: for any $\mathbf{A} \in \mathcal{V}$ and $a, b \in A$

$$
a \approx \approx_{\mathrm{A}} b \text { if and only if } e(a, b)=0 .
$$

In the second case however (Lemma 4.3), the relation $\preccurlyeq_{\mathbf{A}}$ is term-definable: for $\mathbf{A} \in \mathscr{V}$ and $a, b \in A$

$$
a \preccurlyeq_{\mathrm{A}} b \text { if and only if } b * a=0 \text {. }
$$

This is certainly a stronger property and, naturally, it yields a stronger characterization. 
The above remark shows that an investigations of subtractive varieties that have EDPI and also an equivalential term is worthwhile. If a variety $\mathscr{V}$ has an equivalential term, then the principal ideals of $\mathbf{A}$ are closed under dual relative pseudocomplementation (evaluated in the join-semilattice of compact congruences). If $\mathscr{V}$ has EDPI, then the principal ideals of $\mathbf{A}$ are closed under $*$ (that is, the dual relative pseudocomplementation). We shall investigate the conjunction of these two situations, that is, the case in which principal and compact ideals coincide.

A dual Skolem semilattice is an algebra $\langle A, \oplus, \vee, 0\rangle$ such that $\langle A, \vee, 0\rangle$ is a lower bounded join semilattice and for any $a, b, c \in A$,

$$
\begin{array}{ll}
\text { E1 } & a \oplus a=0 ; \\
\text { E2 } & a \oplus b=b \oplus a ; \\
\text { E3 } & a \oplus 0=a ; \\
\text { E4 } & c \vee(a \oplus b)=c \vee((c \vee a) \oplus b) .
\end{array}
$$

Dual Skolem semilattices are equivalent to dual Brouwerian semilattices via the equations

$$
a \oplus b=(a * b) \vee(b * a), \quad a * b=a \oplus(a \vee b)
$$

Hence the variety of dual Skolem semilattices is an ideal determined variety with EDPI. If $\mathbf{A}$ is a Skolem semilattice and $a, b \in A$, then

$$
b \in(a)_{\mathrm{A}} \quad \text { if and only if } a \oplus(a \vee b)=0 .
$$

It is well known [16] that the class of $\{\oplus, 0\}$-subreducts of dual Skolem semilattices is the variety of equivalential algebras and $a \oplus b$ is the dual symmetric relative pseudocomplement of $a$ and $b$. Likewise we have:

LEMMA 4.7. If each two elements $a, b$ of a lower bounded join semilattice have a dual symmetric relative pseudocomplement, then $\langle A, \oplus, \vee, 0\rangle$ is a dual Skolem semilattice.

Now let $\mathbf{A}$ be a subtractive algebra. A join generator [5] for $\mathbf{A}$ is a binary operation $j(x, y)$ such that for any $a, b \in A$

$$
(a)_{\mathbf{A}} \vee(b)_{\mathbf{A}}=(j(a, b))_{\mathbf{A}} .
$$

In general such operation may not be a term and might not even be compatible with the congruences of $\mathbf{A}$ (see the discussion above Theorem 4.3 in [19]). It is also obvious from the definition that if $\mathbf{A}$ has a join generator, then the join of two principal ideals is principal, thus any compact ideal is principal. For a subtractive variety the latter is equivalent to having a join generator term. 
THEOREM 4.8 ([5, Proposition 4.3]). For a subtractive variety $\mathscr{V}$ the following are equivalent:

(1) The join of two principal ideals in any algebra in $\mathscr{V}$ is principal.

(2) Any compact ideal of any algebra in $\mathscr{V}$ is principal.

(3) There is a binary term that is a join generator on any algebra in $\mathscr{V}$.

Let $\mathscr{V}$ a congruence quasi-orderable subtractive variety and suppose that each compact ideal of any algebra in $\mathscr{V}$ is principal. Let $j$ be a join generator term for $\mathscr{V}$ and $s(x, y)$ a subtraction term for $\mathscr{V}$. Define a binary term $e$ on $\mathbf{A} \in \mathscr{V}$ by

$$
e(a, b)=j(s(a, b), s(b, a)) .
$$

Then we can define an operation $\oplus$ on $\mathrm{CI}(\mathbf{A})$,

$$
(a)_{\mathbf{A}} \oplus(b)_{\mathbf{A}}=(e(a, b))_{\mathbf{A}}
$$

and clearly $\langle A, e, \vee, 0\rangle / \approx_{\mathbf{A}} \cong\left\langle\mathrm{CI}(\mathbf{A}), \oplus, \vee,(0)_{\mathrm{A}}\right\rangle$.

THEOREM 4.9. For a subtractive congruence quasi-orderable variety $\mathscr{V}$ the following are equivalent:

(1) For any $\mathbf{A} \in \mathscr{V}$ the join of two principal ideals of $\mathbf{A}$ is principal.

(2) There are binary terms $e$ and $j$ such that, for any $\mathbf{A} \in \mathscr{V},\langle A, e, j, 0\rangle / \approx_{\mathbf{A}}$ is a dual Skolem semilattice.

PROOF. By Lemma 4.7, to show that (1) implies (2) we have only to prove that $e(x, y)$, as defined in (4.1), is the dual symmetric relative pseudocomplementation on $\left\langle\mathrm{CI}(\mathbf{A}), \vee,(0)_{\mathbf{A}}\right\rangle$. Since $\operatorname{PI}(\mathbf{A})=\mathrm{CI}(\mathbf{A})$ this amounts to showing that $e(x, y)$ is an equivalential term for $\mathscr{V}$. By definition of $e$,

$$
e(a, 0)=j(a, s(0, a))=e(0, a) .
$$

Thus,

$$
(e(a, 0))_{\mathbf{A}}=(e(0, a))_{\mathbf{A}}=(j(a, s(0, a)))_{\mathbf{A}}=(a)_{\mathbf{A}} \vee(s(0, a))_{\mathbf{A}}=(a)_{\mathbf{A}},
$$

since $s(0, a) \in(a)_{\mathrm{A}}$. By Theorem 3.2 (4), $\oplus$ is an equivalential term for $\mathscr{V}$.

Conversely assume $(2)$ and note that $j(0,0) / \approx_{\mathbf{A}}=j\left(0 / \approx_{\mathbf{A}}, 0 / \approx_{\mathbf{A}}\right)=0 / \approx_{\mathbf{A}}$, implying that $e(0,0)=0$ holds in A. Next

$$
j(a, b) \vartheta_{\mathbf{A}}(a, 0) \vee \vartheta_{\mathbf{A}}(b, 0) j(0,0)=0,
$$

so $j(a, b) \in(a)_{\mathbf{A}} \vee(b)_{\mathbf{A}}$. Finally from $j(a, b) / \approx_{\mathbf{A}}=j\left(a / \approx_{\mathbf{A}}, b / \approx_{\mathbf{A}}\right)$ we get that $a / \approx_{\mathbf{A}} \in\left((j(a, b)) / \approx_{\mathbf{A}}\right)_{\mathbf{A} / \approx_{\mathrm{A}}}$. Thus Corollary 2.2 yields

$$
a \in(j(a, b))_{\mathrm{A}} \vee 0 / \approx_{\mathrm{A}}=(j(a, b))_{\mathrm{A}} .
$$


Symmetrically $b \in(j(a, b))_{\mathbf{A}}$ and in conclusion $(a)_{\mathbf{A}} \vee(b)_{\mathbf{A}}=(j(a, b))_{\mathbf{A}}$. So $j(x, y)$ is a join generator term for $\mathbf{A}$ and the join of any two principal ideals is principal.

We separate the consequences of Theorem 4.9 into distinct corollaries. The first one generalizes [6, Theorem 3.9].

COROLLARY 4.10. A subtractive congruence quasi-orderable variety in which the join of two principal ideals is principal has a quasi-Mal'cev term.

COROLLARY 4.11. A subtractive congruence quasi-orderable variety in which the join of two principal ideals is principal has EDPI.

COROLLARY 4.12. If $\mathscr{V}$ is a subtractive congruence quasi-orderable variety in which the join of two principal ideals is principal, then $\mathscr{V}_{\varepsilon}$ is a congruence orderable variety.

We can apply Theorem 4.5 to conclude any subtractive congruence quasi-orderable variety in which the join of two principal ideals is principal is termwise equivalent to a variety of MINI algebras with compatible operations. The reader can easily check that, if $\mathscr{V}$ is such a variety and $\mathbf{A} \in \mathscr{V}$, then for $a, b \in \mathbf{A}$

$$
b \in(a)_{\mathbf{A}} \text { if and only if } e(a, j(a, b))=0 \text {, }
$$

hence the term $x * y=e(x, j(x, y))$ is the MINI algebra operation. Determining the structure of $\langle A, e, j, 0\rangle$ for any algebra $\mathbf{A} \in \mathcal{V}$ can be done in a similar way to the one used for going from dual Hilbert algebras to MINI algebras.

Let $\mathscr{B}$ be the variety of algebras $\mathbf{A}=\langle A, *, j, 0\rangle$ where $\langle A, *, 0\rangle$ is a MINI algebra and moreover for any $a, b, c \in A$,

(B1) $j(0,0)=0$;

(B2) $(c * a) *((c * b) *(c * j(a, b)))=0$;

(B3) $j(a, b) * b=j(a, b) * a=0$.

It is an easy but tedious exercise to check that (B2) and (B3) ensure that $j(x, y)$ is a compatible operation with the MINI algebra structure so that $\operatorname{Con}(A)=\operatorname{Con}(\langle A, *, 0\rangle)$. This implies that $\approx_{\mathbf{A}} \in \operatorname{Con}(\mathbf{A})$. Moreover, (B3) and (B1) imply that $j(x, y)$ is a join generator term for $\mathbf{A}$. Hence $\mathscr{B}$ is a subtractive congruence quasi-orderable variety in which the join of two principal ideals is principal.

THEOREM 4.13. A pointed variety is congruence quasi-orderable, subtractive and the join of two principal ideals is principal if and only if it is termwise equivalent to a variety of $\mathscr{B}$-algebras with compatible operations (as usual compatible with the MINI algebra structure). 
PROOF. One half of the theorem has been already proved. Conversely if $\mathscr{V}$ is such a variety, then by Theorem 4.5 , has EDPI and hence there is a binary term * such that for any $\mathbf{A} \in \mathscr{V},\langle A, *, 0\rangle$ is a MINI algebra. Next by Theorem 4.8, $\mathscr{V}$ has a join generator $j(x, y)$. Again by Theorem 4.5 (and from the fact that dual Skolem semilattices and dual Brouwerian semilattices are definitionally equivalent) $\langle A, *, j, 0\rangle / \approx_{\mathrm{A}}$ is a dual Brouwerian semilattice. It follows that if $t\left(x_{1}, \ldots, x_{n}\right)$ is a term in the language $\{*, j, 0\}$ and if $t\left(x_{1}, \ldots, x_{n}\right) \approx 0$ holds in any dual Brouwerian semilattice, then $t\left(x_{1}, \ldots, x_{n}\right) \approx 0$ holds in $\mathscr{V}$. But (B1), (B2) and (B3) all hold in Brouwerian semilattices, hence $\langle A, *, j, 0\rangle \in \mathscr{B}$. That any basic operation of $\mathbf{A}$ is compatible is obvious.

Since Brouwerian semilattices are simply congruence orderable $\mathscr{B}$-algebras we get as a corollary the result in [15] that any congruence orderable arithmetical variety is termwise equivalent to a variety of Brouwerian semilattices with compatible operations. Let us go further in our investigation. While compact ideals are always closed under joins they are not in general closed under meets. We take a closer look at this case.

If $\mathbf{A}$ is a subtractive algebra, a meet generator [5] for $\mathbf{A}$ is a binary operation $m(x, y)$ such that for any $a, b \in A$

$$
(a)_{\mathbf{A}} \cap(b)_{\mathbf{A}}=(m(a, b))_{\mathbf{A}} .
$$

If a subtractive variety has a meet generator term then clearly the principal ideals are closed under meets. The vice versa, however, is not true unless we assume ideal distributivity.

THEOREM 4.14 ([5, Theorem 4.2]). For a subtractive variety $\mathscr{V}$ the following are equivalent:

(1) $\mathscr{V}$ has a meet generator term.

(2) $\mathscr{V}$ is ideal distributive and the meet of two principal ideals of any algebra in $\mathscr{V}$ is principal.

Hence if in a congruence quasi-orderable subtractive variety $\mathscr{V}$ the principal ideals are closed under joins and meets, then $\mathscr{V}$ has EDPI (and hence it is ideal distributive). So $\mathscr{V}$ has both a meet generator term $m$ and a join generator term $j$ and $\left\langle\mathrm{PI}(\mathrm{A}), *, \vee, \cap,(0)_{\mathrm{A}}\right\rangle$ is a (dual) relatively pseudocomplemented lattice, isomorphic with $\langle A, *, j, m, 0\rangle / \approx_{\mathrm{A}}$.

A representation result for such kind of varieties can be obtained now very easily. Let $\mathscr{L}$ be the variety of algebras such that $\mathbf{A}=\langle A, *, j, m, 0\rangle$ where $\langle A, *, j, m, 0\rangle \in$ $\mathscr{B}$ and moreover for any $a, b, c \in A$

(L1) $m(0,0)=0$; 
(L2) $(a * c) *((b * c) *(m(a, b) * c))=0$;

(L3) $a * m(a, b)=b * m(a, b)=0$.

By the same argument we have used over and over in this paper, one sees that the variety of $\mathscr{L}$-algebras is a congruence quasi-orderable subtractive variety in which principal ideals are closed under joins and meets. Likewise we have:

THEOREM 4.15. A pointed variety is congruence quasi-orderable subtractive and the principal ideals are closed under joins and meets if and only if it is termwise equivalent to a variety of $\mathscr{L}$-algebras with compatible operations.

Since relatively pseudocomplemented lattices are simply congruence orderable $\mathscr{L}$-algebras we get:

COROLLARY 4.16. A pointed variety is congruence orderable, subtractive and the principal ideals are closed under joins and meets if and only if it is termwise equivalent to a variety of relatively pseudocomplemented lattices with compatible operations.

The lattice of subvarieties of the variety $\mathscr{R}$ of relative Stone lattices is completely known $[14,18]$. It is a countable chain with a top element and the $n$-th level variety $\mathscr{R}_{n}$ is just the subvariety of $\mathscr{R}$ generates by a chain of $n$ elements. $\mathscr{R}_{n}$ can be axiomatized, relative to $\mathscr{R}$, by the single equation

$$
\left(x_{1} * x_{2}\right) \wedge\left(x_{2} * x_{3}\right) \wedge \ldots \wedge\left(x_{n} * x_{n+1}\right)=0
$$

Hence the variety $\mathscr{R}_{2}$ is just the variety of generalized Boolean algebras.

The form of the above equation allows us to conclude the following. A congruence quasi-orderable subtractive variety $\mathscr{V}$ in which principal ideals are closed under joins and meets is such that for any $\mathrm{A} \in \mathscr{V},\langle A, \vee, \wedge, *, 0\rangle / \approx_{\mathrm{A}} \in \mathscr{R}_{n}$ if and only if $R_{n}$ holds in $\mathscr{V}$. In [5, Example 5.9] it is basically shown that the variety of pseudocomplemented semilattices is of this kind. It fact it satisfies $R_{2}$ and hence the (principal) ideals form a Boolean algebra. Since the variety of pseudocomplemented semilattices is not congruence distributive, it is a further example of a congruence quasi-orderable variety that is not congruence orderable.

\section{References}

[1] P. Agliano, 'Fregean subtractive varieties with definable congruences', J. Austral. Math. Soc. 71 (2000), 353-366.

[2] P. Agliano and A. Ursini, 'Ideals and other generalizations of congruence classes', J. Austral. Math. Soc. Ser. A 53 (1992), 103-115.

[3] —-, 'On subtractive varieties II: general properties', Algebra Universalis 36 (1996), 222-259. 
[4] _ _ 'On subtractive varieties III: From ideals to congruences', Algebra Universalis 37 (1997), $296-333$.

[5] —_, 'On subtractive varieties IV: definability of principal ideals', Algebra Universalis 38 (1997), 355-389.

[6] W. J. Blok, P. Köhler and D. Pigozzi, 'On the structure of varieties with equationally definable principal congruences II', Algebra Universalis 18 (1984), 334-379.

[7] W. J. Blok and D. Pigozzi, 'Algebraic semantics for universal horn logic without equality', in: Universal algebra and quasi-group theory (eds. A. Romanowska and J. H. D. Smith) (Heldermann, Berlin, 1992) pp. 1-56.

[8] W. J. Blok and J. G. Raftery, 'Ideals in quasivarieties of algebras', in: Models, algebras and proofs (eds. X. Caceido and C. H. Montenegro), Lecture Notes in Pure and Appl. Math. 203 (Marcel Dekker, New York, 1999) pp. 167-186.

[9] J. R. Büchi and M. T. Owens, 'Complemented monoids and hoops', unpublished manuscript.

[10] J. Czelakowski and D. Pigozzi, 'Fregean algebraic logic', preprint.

[11] _- 'Fregean logics with the multiterm deduction theorem and their algebrization', preprint.

[12] J. Font, 'On the Leibniz congruences', in: Algebraic methods in logic an computer science (ed. C. Rauszer), Banach Center Publ. 28 (Polish. Acad. Sci., Warszawa, 1993) pp. 17-36.

[13] J. Font and R. Jansana, A general semantics for sentential logics, Lecture Notes in Logic 7 (Springer, New York, 1996).

[14] T. Hecht and T. Katrinak, 'Equational classes of relative Stone algebras', Notre Dame J. Formal Logic 13 (1972), 248-254.

[15] P. M. Idziak, K. Słomczyńska and A. Wroński, 'Equivalential algebras: a study of Fregean varieties', preprint, 1996.

[16] J. K. Kabziński and A. Wroński, 'On equivalential algebras', in: Proceedings of the 1975 International Symposium om Multiple-Valued Logic (Bloomington, Ind., 1975) (IEEE Comput. Soc., Long Beach, CA. 1975) pp. 419-428.

[17] P. Köhler and D. Pigozzi, 'Varieties with equationally definable principal congruences', Algebra Universalis 11 (1980), 213-219.

[18] W. Nemitz and T. Whaley, 'Varieties of implicative semilattices', Pacific J. Math. 37 (1971), 759-769.

[19] D. Pigozzi, 'Fregean algebraic logic', in: Algebraic logic (eds. H. Andréka, J. D. Monk and I. Németi), Colloq. Math. Soc. János Bolyai 54 (North-Holland, Amsterdam, 1991) pp. 473-502.

[20] A. Ursini, 'On subtractive varieties I', Algebra Universalis 31 (1994), 204-222.

[21] A. Wroński, 'BCK-algebras do not form a variety', Math. Japon. 28 (1983), 211-213.

\section{Dipartimento di Matematica}

Via del Capitano 15

53100, Siena

Italy

e-mail: agliano@unisi.it 
